\title{
Vermittlung einer Krebsdiagnose, eine individuelle Herausforderung
}

Onkologen haben ein erhebliches Burnout-Risiko, auch wegen der großen Belastung bei der Übermittlung schlechter Nachrichten. Wie sie dieser schwierigen Aufgabe begegnen, wurde in einer Metasynthese untersucht.

ᄃ inem Patienten unangenehme NachE richten zu übermitteln, gehört nach wie vor zu den größten Herausforderungen für Onkologen. Wie Ärzte diesen schwierigen Momenten zu begegnen

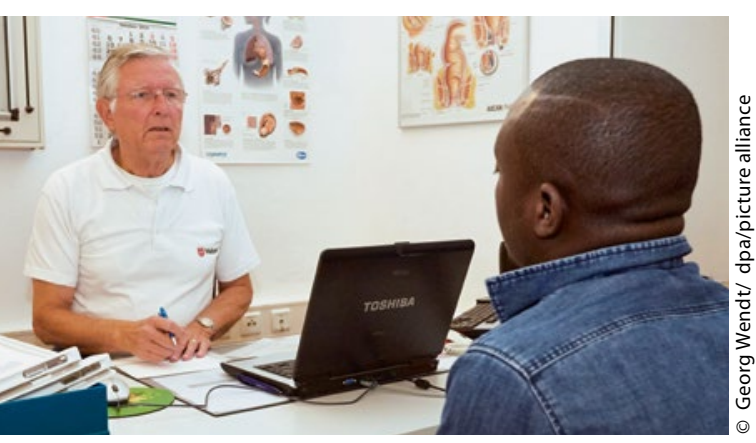

Schulungen erwünscht: Wie schlechte Nachrichten an Menschen aus anderen Kulturkreisen überbringen? versuchen, haben französische Kollegen anhand einer Literaturanalyse untersucht. Insgesamt wurden 40 hochwertige Artikel gefunden, die 600 Onkologen aus 12 Ländern verfasst hatten.

Wichtigstes Thema war die Begegnung von Arzt und Patient mitsamt ihren kommunikativen Aspekten beim Übermitteln der schlechten Nachrichten und dem Umgang mit den auftretenden Emotionen. Ein weiteres Thema waren externe Faktoren, die die Arzt-Patienten-Beziehung beeinflussten, darunter die Familie des Patienten sowie die systemischen, institutionellen und kulturellen Faktoren.

Die meisten Onkologen waren sich der Problematik der Situation für ihre Patienten bewusst. Sie versuchten, den Patienten in seiner sozialen Situation zu betrachten und herauszufinden, was die
Nachricht für ihn bedeuten würde, bevor sie sie übermittelten. Die Nachricht beeinflusste aber auch die Onkologen in ihren eigenen Empfindungen. Dabei nutzten sie sehr unterschiedliche Copingstrategien - emotionsbezogene, sachbezogene und sinnbezogene. In allen Studien äußerten die Onkologen größere Probleme, wenn sie schlechte Nachrichten an Menschen aus anderen Kulturkreisen übermitteln sollten und wünschten sich mehr Ausbildung in diesem Bereich.

Fazit: Bedeutende negative Nachrichten sind ein Balanceakt für Onkologen, in dem sie sich noch dazu an individuell sehr unterschiedliche äußere Faktoren anpassen müssen. Es wäre ein großer Schritt nach vorn, wenn sie in ihrer Ausbildung lernten, die Gesprächsführung an individuell unterschiedliche Bedingungen anzupassen.

Christina Berndt

Bousquet $\mathrm{G}$ et al. Breaking Bad News in Oncology: A Metasynthesis. J Clin Oncol. 2015;33(22):2437-43.

\section{Versorgung am Lebensende: Mehr aggressive Therapien in der Hämatologie}

Um Entscheidungen im Hinblick auf die Versorgung am Lebensende des Krebspatienten besser verstehen zu können, wurden die Empfehlungen zur Tumortherapie und die Einstellung zur palliativen Versorgung bei Hämatologen und Spezialisten für solide Tumoren verglichen.

B efragt wurden insgesamt 120 Hämatologen und 120 Spezialisten für solide Tumoren. Die Ärzte beantworteten einen Fragebogen zu verschiedenen Aspekten der Versorgung am Lebensende anhand von drei standardisierten Fallbeispielen, z. B. zur palliativen systemischen Therapie und zu Kenntnissen in der palliativen Versorgung.

$76 \%$ der befragten Ärzte gaben Auskunft. Im Vergleich zu Spezialisten für solide Tumoren favorisierten Hämatologen auch noch bei Patienten mit einem ECOG-Performancestatus von 4 und einem erwarteten Überleben von nur einem Monat signifikant häufiger die Verordnung einer systemischen Therapie mit moderater Toxizität und ohne Nutzen hinsichtlich des Überlebens (mediane Präferenz 4 vs. 1 , wobei $1=$ sehr gegen die Therapie und $7=$ sehr für die Therapie bedeutet; $\mathrm{p}<0,0001)$.

Darüber hinaus fühlten sich Hämatologen bei der Diskussion um Tod und Sterben ( 72 vs. $88 \%$; $p=0,007)$ und bei Überweisungen in ein Hospiz (81 vs. $93 \%$; $\mathrm{p}=0,02$ ) weniger wohl als Onkologen, die sich auf solide Tumoren spezialisiert hatten. Auch hatten die befragten Hämatologen häufiger das Gefühl des Versagens, wenn sie den Krankheitsverlauf nicht beeinflussen konnten (46vs. $31 \%$; $\mathrm{p}=0,04$ ). Etwas mehr als die Hälfte der befragten Ärzte fühlte sich zufrieden, wenn sie sterbenden $\mathrm{Pa}$ tienten eine „End-of-life-Versorgung“ anbieten konnte.

In der multivariaten Analyse waren das Vorliegen einer hämatologischen Krebserkrankung (Odds Ratio [OR] 2,77; $\mathrm{p}=0$ 0,002) und das Ausmaß des "sich wohlfühlens“ mit der Therapieverordnung (OR 3,79; $\mathrm{p}=0$,02) damit assoziiert, welche Therapie in den letzten Lebensmonaten verordnet wurde.

Fazit: In der Studie wurden signifikante Unterschiede in den Einstellungen und Überzeugungen bezüglich der Versorgung der Patienten am Lebensende zwischen Hämatologen und Spezialisten für solide Tumoren aufgedeckt.

Judith Neumaier

Hui $D$ et al. Differences in attitudes and beliefs toward end-of-life care between hematologic and solid tumor oncology specialists. Ann Oncol. 2015;26(7):1440-6. 